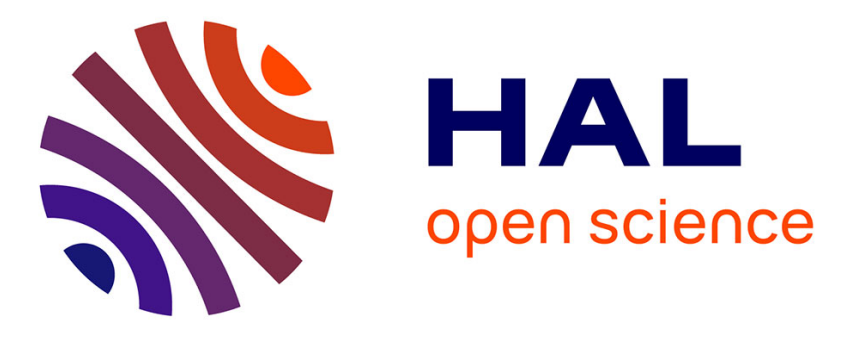

\title{
Self-consistent estimates for nonlinear viscoelastic polycrystals: a simplified resolution of the affine formulation adapted to monotonic loading paths
}

Rénald Brenner, Olivier Castelnau, Renaud Masson, Brigitte Bacroix, André Zaoui

\section{To cite this version:}

Rénald Brenner, Olivier Castelnau, Renaud Masson, Brigitte Bacroix, André Zaoui. Self-consistent estimates for nonlinear viscoelastic polycrystals: a simplified resolution of the affine formulation adapted to monotonic loading paths. Key Engineering Materials, 2002, 177-180, pp.153-158. 10.4028/www.scientific.net/KEM.177-180.153 . hal-00111367

\author{
HAL Id: hal-00111367 \\ https://hal.science/hal-00111367
}

Submitted on 29 Jul 2019

HAL is a multi-disciplinary open access archive for the deposit and dissemination of scientific research documents, whether they are published or not. The documents may come from teaching and research institutions in France or abroad, or from public or private research centers.
L'archive ouverte pluridisciplinaire HAL, est destinée au dépôt et à la diffusion de documents scientifiques de niveau recherche, publiés ou non, émanant des établissements d'enseignement et de recherche français ou étrangers, des laboratoires publics ou privés.

\section{(c)(1)}

Distributed under a Creative Commons Attribution| 4.0 International License 


\title{
Self-consistent Estimates for Nonlinear Viscoelastic Polycrystals: a Simplified Resolution of the Affine Formulation Adapted to Monotonic Loading Paths
}

\author{
R. Brenner ${ }^{1.2}$, O. Castelnau', R. Masson ${ }^{3}$, B. Bacroix ${ }^{1}$ and A. Zaoui ${ }^{3}$ \\ 'LPMTM-CNRS, Inst. Galilée, Univ. Paris, 13, av. J.B. Clément, FR-93430 Villetaneuse, France \\ ${ }^{2}$ Centre de Saclay, CEA-CEREM / SRMA, FR-91191 Gif-sur-Yvette, France \\ ${ }^{3}$ LMS. Ecole Polytechnique, FR-91128 Palaiseau Cedex, France
}

Keywords: Non Linear Viscoelasticity, Polycrystals, Self-Consistent Scheme

\begin{abstract}
A simplified resolution of the self-consistent model for non-linear viscoclastic polycrystals is presented. The formulation is based on an affine linearization of the constitutive relations as recently proposed by Masson and Zaoui [4], which adapts Hill's conception of self-consistent modelling to nonlinear viscoelastic behaviours. The approximation of the inverse Laplace-Carson transform introduced here, which is believed to be well adapted to monotonic loading paths, makes the resolution of the whole self-consistent problem possible in the direct time space. This results in a largely simplified numerical algorithm. Comparisons with the predictions of a model treating accurately the inverse Laplace-Carson transform arc presented, and very good results are obtained for creep of FCC and HCP polycrystals.
\end{abstract}

\section{INTRODUCTION}

In the frame of the multiscale modelling of heterogeneous materials (in particular polycrystals), several propositions were made during the last twenty years in view of estimating the overall response of nonlinear viscoelastic (or elastoviscoplastic) materials. The specific difficulty of the problem, as compared to the case of elasticity or viscoplasticity, is that the response of the material at a given time $\tau$ depends on the whole thermomechanical history for time $t \in[-\infty ; \tau[$ owing to the viscoelastic coupling. That is, the linearized behaviour has to be defined carefully enough in order to save the viscoelastic character of the interactions between the phases. The first attempt to deal with this problem was proposed by Weng [13]. By considering the viscoplastic strain as a stressfree strain (in the sensc of Eshelby), this model leads to (strong) elastic interactions between the different phases; this treatment is similar to that proposed by Kröner for elastoplasticity. Different approaches based on a postulated interaction equation have been proposed. The non-incremental formulation proposed by Molinari et al. [6] consists in considering a Maxwell-type behaviour at both the microscopic and macroscopic scales. Such an assumption neglects the "long range memory effect " and consequently, the viscoelastic character of the interactions is only partially retained. Turner et al. [12] proposed an incremental scheme with an interaction equation tuned in such a way that the limit steady state behaviour $(t \rightarrow+\infty)$ corresponds to the one described by the tangent viscoplastic self-consistent model [7]. This model is limited to a power-law viscoplastic potential with the same stress exponent $n$ for all the slip systems. An approach with internal variables was recently proposed by Paquin [8] and, for that goal, the self-consistent scheme had to be modified.

Recently, Masson and Zaoui [4] showed that Hill's basic conception of the non-linear selfconsistent modelling still holds for nonlinear viscoelastic polycrystals. They derived an affine (non incremental) formulation which retains entircly the viscoelastic nature of the interactions and 
showed that the viscoelastic problem is equivalent to a symbolic thermo-elastic one in the LaplaceCarson space. This formulation does not require any particular assumption on the local viscoplastic potential (except convexity) and the prediction of the steady-state regime is found to lie between the secant and the tangent viscoplastic ones [3] (this latter can be considered as an approximation of the affine model [5]). Although tractable, the nature of the constitutive relations renders the numerical resolution quite complex and time-consuming. Based on the same affine formulation, we propose a simplified resolution of the problem for the particular case of monotonic loading paths. The approximation of the inverse Laplace-Carson transform introduced here allows the resolution of the whole self-consistent problem in the direct time space.

\section{FORMULATION}

\subsection{Linearization of the local constitutive law}

We consider a polycrystal whose phases present a Maxwell type behaviour with a non linear viscous part. The local constitutive law can thus be written

$$
\dot{\boldsymbol{\varepsilon}}(\mathrm{t})=\mathbf{m}: \dot{\boldsymbol{\sigma}}(\mathrm{t})+\dot{\boldsymbol{\varepsilon}}^{v p}(\mathrm{t})
$$

with $\mathbf{m}$ the elastic compliance tensor and $\dot{\boldsymbol{\varepsilon}}^{\mathrm{vp}}(\mathfrak{l})$ the viscoplastic strain rate deriving from a strictly convex potential $\omega$

$$
\dot{\varepsilon}^{v p}(t)=\frac{\partial \omega}{\partial \sigma}(\sigma(t))=g(\sigma(t))
$$

In the following, we do not consider the dependence of $\dot{\varepsilon}^{\text {n) }}(t)$ on internal variables such as intracrystalline hardening, whereas this can be introduced with no major difficulties.

In order to follow the homogenization schemes developed in a linear frame, it is necessary to use a linearized expression of (1). Due to the nature of the constitutive equations $(\dot{\varepsilon}=f(\boldsymbol{\sigma}, \boldsymbol{\sigma}))$, the definition of a classical tangent modulus as for elastoplasticity or viscoplasticity is no more possible. Although we want to determine the macroscopic responsc of the polycrystal at a given time $t=\tau$ in a non-incremental way, the definition of the linearized local law at time $\tau$ is not sufficient. Indeed, due to the viscoelastic coupling, it is nccessary to know in addition the local strain rate for any times $t$ up to $\tau$. To do so, we use the linearization procedure proposed by Rougier et al. [9]. The local constitutive relation at time $t$ and linearized at time $\tau$ reads

$$
\begin{aligned}
\dot{\varepsilon}(t, \tau)=m & : \dot{\sigma}(t)+\mathbf{k}(\tau): \sigma(t)+\dot{\varepsilon}_{0}(\tau) \quad \text { for any } t \geq \tau \\
\dot{\varepsilon}_{0}(\tau)=g(\sigma(\tau))-\mathbf{k}(\tau): \sigma(\tau) & \\
\mathbf{k}(\tau)=\frac{\mathrm{dg}}{\mathrm{d} \sigma} \sigma(\tau) &
\end{aligned}
$$

For any time $t<\tau$, we keep the exact (non linearized) expression of the local law (1). In order to have a expression similar to (3) for any time $t \in[0 ;+\infty[$, the local constitutive law is taken as

$$
\begin{gathered}
\dot{\varepsilon}(t, \tau)=\mathbf{m}: \dot{\sigma}(t)+\mathbf{k}(\tau) ; \sigma(t)+\dot{\varepsilon}_{0}(t, \tau) \quad \text { for any } t \in[0 ;+\infty[ \\
\dot{\varepsilon}_{0}(t, \tau)=g(\sigma(\tau))-k(\tau): \sigma(\tau)+[g(\sigma(t))-g(\sigma(\tau))-k(\tau):(\sigma(t)-\sigma(\tau))][1-H(t-\tau)]
\end{gathered}
$$

with $H(t)$ the unit step function. The integration of equations (4) leads to

$$
\begin{gathered}
\boldsymbol{\varepsilon}(\mathrm{t}, \tau)=\left(\mathbf{m}_{v e} * \boldsymbol{\sigma}\right)(\mathrm{t}, \tau)+\boldsymbol{\varepsilon}_{0}(\mathrm{t}, \tau) \\
\mathbf{m}_{\mathrm{ve}}(\mathrm{t}, \tau)=\mathbf{m}+\mathbf{k}(\tau) \mathrm{t}
\end{gathered}
$$




$$
\varepsilon_{0}(\mathrm{t}, \tau)=\int_{0}^{1} \dot{\varepsilon}_{0}(\mathrm{u}, \tau) \mathrm{du}
$$

where $*$ denotes the convolution product in the sense of Stieltjes. Thus, for any linearization time $\tau$ we have to solve a linear viscoelastic problem with eigenstrains.

\subsection{Laplace-Carson transform}

The resolution of this problem is classically performed using the correspondence principle proposed by Laws and McLaughlin [1] which allows to convert the linear viscoelastic problem into a symbolic linear elastic problem in the Laplace-Carson space. Equation (5) gives

$$
\begin{gathered}
\hat{\boldsymbol{\varepsilon}}(\mathrm{p}, \tau)=\hat{\mathbf{m}}_{\mathrm{ve}}(\mathrm{p}, \tau): \hat{\boldsymbol{\sigma}}(\mathrm{p})+\hat{\boldsymbol{\varepsilon}}_{\mathrm{o}}(\mathrm{p}, \tau) \\
\hat{\mathbf{m}}_{\mathrm{ve}}(\mathrm{p}, \tau)=\mathbf{m}+\frac{1}{\mathrm{p}} \mathbf{k}(\tau)
\end{gathered}
$$

where the transform $\hat{f}(p)$ of a function $f(t)$ is defined by

$$
\hat{f}(p)=p \int_{0}^{+\infty} f(t) e^{\cdot p t} d t
$$

\subsection{Self-consistent scheme}

In order to derive the overall properties of the polycrystal, we apply the self-consistent scheme in its classical form. Each phase (grains with the same crystallographic orientation) is treated as an ellipsoidal inhomogeneity embedded in the homogeneous effective medium (HEM). In the frame of small dcformations, we do not take into account the crystallographic and morphological texture evolution. The homogenization scheme leads to the following usual equations defining the overall modulus and eigenstrain

$$
\begin{aligned}
& \hat{\mathbf{M}}_{\text {ve }}(p, \tau)=\left\langle\hat{\mathbf{m}}_{\text {ve }}(p, \tau): \hat{\mathbf{B}}(p, \tau)\right\rangle \\
& \hat{\mathbf{E}}_{0}(p, \tau)=\left\langle\hat{{ }^{i}} \mathbf{B}(p, \tau): \hat{\boldsymbol{\varepsilon}}_{0}(p, \tau)\right\rangle
\end{aligned}
$$

$\hat{\mathbf{B}}(\mathrm{p}, \tau)$ is the stress concentration tensor; it depends on the modulus of the inhomogeneity $\hat{\mathbf{m}}_{\mathrm{vc}}(\mathrm{p}, \tau)$ and of the HEM $\hat{\mathbf{M}}_{\mathrm{ve}}(\mathrm{p}, \tau)$, and also on the shape and orientation of the inhomogeneity via the Eshelby tensor. Equation (8) must be solved iteratively owing to its implicit character.

\subsection{Inversion of the Laplace-Carson transforms}

Once the problem is solved in the Laplace-Carson space, it is necessary to proceed to the inversion of the result in order to get the overall properties in the direct time space $\left(\mathbf{M}_{\mathrm{ve}}(\mathrm{t}, \tau)\right.$ and $\left.\mathrm{E}_{0}(\mathrm{t}, \tau)\right)$. Two efficient numerical methods have been proposed by Schapery [10] for that purpose. One of them (the collocation method) has been successfully applied in previous works $[4,11]$. It is based on an approximation of the function $\mathrm{f}(\mathrm{t})$ by a Dirichlet serie. The choice of $N$ collocation points $\rho$, which have to be adjusted carefully, involves $N$ resolutions of the self-consistent equations (8). The advantage of this method is that the inverse transform can always been made more accurately by increasing the value of $N$. Although tractable, this method renders the implementation of the affine model in non linear viscoelasticity quite complex. Here, we propose to use a direct evaluation of the function $f(t)$ (the direct method). According to Schapery, if a function $f(t)$ presents locally a quasi- 
linear behaviour versus $\log (t)$ for at least two decades of $t, \hat{f}(p)$ and $f(t)$ are directly connected by the relation

$$
f(t) \approx \hat{f}(p)_{p=1 / 2 t}
$$

For the application to our problem, the different variables which have to fulfill the above condition are the stresses, the strains and the moduli at the local and macroscopic scales. It is believed that it can reasonably be used for any monotonic loading path (creep, relaxation, constant strain rate test...). As already shown by Turner et al. [12], the direct method gives very satisfactory results for linear viscoelasticity. Its main drawback, as compared to the collocation method, is that the accuracy of the inverse transform can not be increased.

Applied to the resolution of the affine model, the direct inverse transform implies that the symbolic thermoelastic problem in the Laplace-Carson transform holds in the direct time space. To obtain the overall response at time $t=\tau$, the affine model can thus be written

At the local scale :

$$
\begin{aligned}
& \boldsymbol{\varepsilon}(\tau)=\mathbf{m}_{\mathrm{ve}}(\tau): \boldsymbol{\sigma}(\tau)+\boldsymbol{\varepsilon}_{0}(\tau) \\
& \qquad \boldsymbol{\varepsilon}_{0}(\tau)=\int_{0}^{\tau} \dot{\boldsymbol{\varepsilon}}_{0}(\mathrm{t}, \tau) \mathrm{dt} \\
& \text { with } \dot{\boldsymbol{\varepsilon}}_{0}(\mathrm{t}, \tau)=g(\boldsymbol{\sigma}(\mathrm{t}))-\mathbf{k}(\tau): \boldsymbol{\sigma}(\mathrm{t}) \quad \text { for any } t \in[0 ; \tau] \\
& \text { and } \mathbf{m}_{\mathrm{ve}}(\tau)=\mathbf{m}+\mathbf{k}(\tau) \tau
\end{aligned}
$$

At the macroscopic scale : $\quad \mathrm{E}(\tau)=\mathbf{M}_{\mathrm{ve}}(\tau): \Sigma(\tau)+\mathrm{E}_{0}(\tau)$

Interaction law : $\quad \boldsymbol{\varepsilon}(\tau)-\mathrm{E}(\tau)=-\overline{\mathbf{M}}_{\mathrm{ve}}(\tau):(\boldsymbol{\sigma}(\tau)-\Sigma(\tau))$

Here, the whole thermomechanical history is contained into the term $\dot{\varepsilon}_{0}(t, \tau)$. The use of the direct method thus presents two particularly interesting features :

(i) the numerical resolution of equations (10) can be performed with a simple algorithm similar to that used to solve non-linear viscoplastic problems [2].

(ii) the limit behaviour at $\mathrm{t}=0$ and $\mathrm{t} \rightarrow+\infty$ is purely elastic and purely viscoplastic respectively and is strictly similar to that obtained when using the more accurate collocation method.

Note that if instead of the expression used here, the stress-free strain rate is supposed to be constant for any $t \in[0 ; \tau]$ and equal to his value at time $t=\tau$, i.e.

$$
\dot{\varepsilon}_{0}(t, \tau)=\dot{\varepsilon}_{0}(\tau)=g(\sigma(\tau))-\mathbf{k}(\tau): \sigma(\tau)
$$

then, the linearized viscoelastic problem degenerates into an elastic problem with the viscoplastic strain considered as a stress-free strain so that the mechanical history is ignored. This is nothing else but the model leading to elastic interactions as proposed by Weng.

\section{APPLICATIONS}

The numerical code we have developed fully takes into account the anisotropy of the elastic and plastic behaviours at both microscopic and macroscopic scales. The calculation time of a mechanical test requires approximately 10 minutes on an ordinary PC, and convergence is obtained for high non-linearities of the viscoplastic behaviour (up to $n=30$ with a power law potential).

As a first application, we present comparisons between the resolution of the affine formulation by use of the more accurate collocation method [4] and the direct method as proposed here, for nonlinear creep. We use a local power law viscoplastic potential 


$$
\omega(\boldsymbol{\sigma})=\sum_{s} \frac{\dot{\gamma}_{0}^{s} \tau_{0}^{s}}{n+1}\left|\frac{\mathbf{R}^{s}: \boldsymbol{\sigma}}{\tau_{0}^{s}}\right|^{n+1}
$$

with $n=5$ and assume local elastic isotropy. We consider FCC and HCP untextured polycrystals with spherical phases. For the FCC case, we assume that viscoplastic strain occurs by glide on octahedral slip systems. For the HCP case, we have considered prismatic, basal and pyramidal $\left\langle c+a>\right.$ slip systems. The respective reference shear stresses $\tau_{0}^{s}$ are indicated in the Figures 1 and 2. These comparisons show a very good agreement of our model with the Masson-Zaoui's predictions; the difference between the two resolutions is about $1 \%$. The steady-state regime described with the scheme proposed is the one of the affine model in viscoplasticity (without internal variables) since

+ collocation method

- direct method

.... elastic interaction scheme

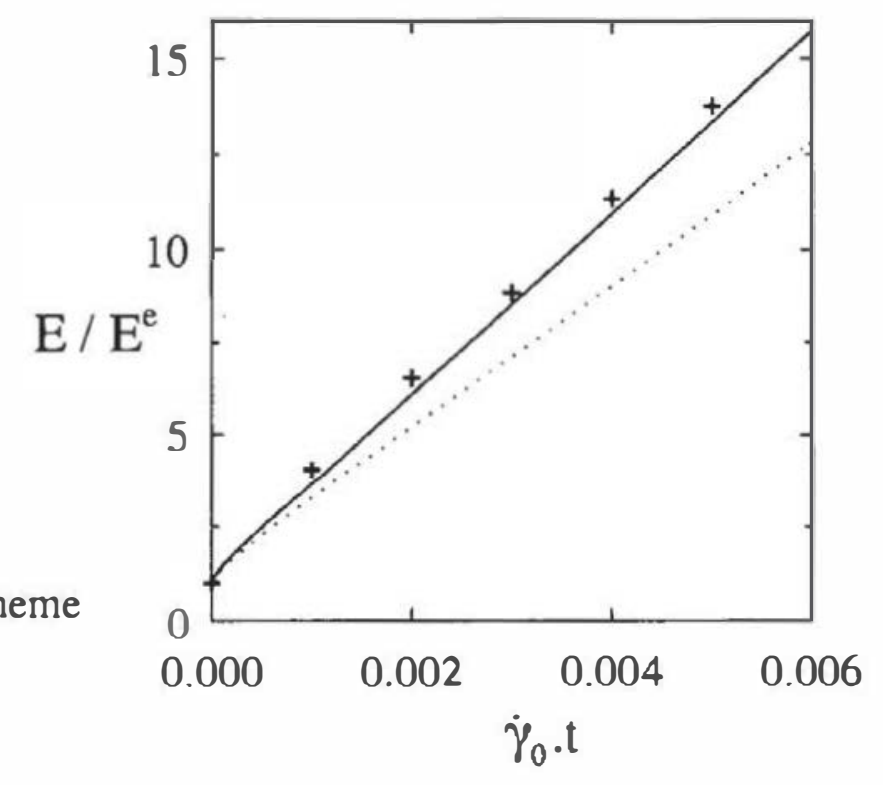

Fig. 1. Evolution of the normalized macroscopic strain during a tensile creep test $\left(\Sigma / \tau_{0}=4\right)$ for a FCC polycrystal without crystallographic texture (Material constants : $\tau_{0} / \mathrm{E}^{\text {Young }}=10^{-3}, v=0.3, \mathrm{n}=5$ )

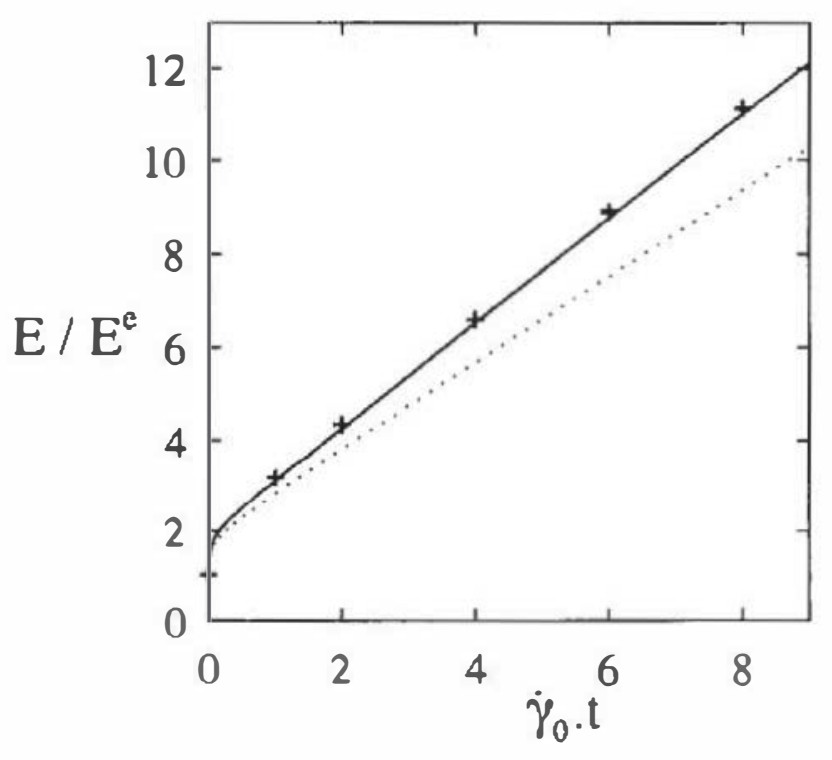

Fig. 2. Evolution of the normalized macroscopic strain during a tensile creep test $\left(\Sigma / \tau_{\text {oppris }}=2\right)$ for a HCP polycrystal without crystallographic texture (Material constants : $2 \tau_{\text {(pris }} / \mathrm{E}^{\text {Young }}=10^{-3}, \nu=0.3, \mathrm{n}=5$, $\tau_{0 \text { (pyrec }+\infty)} / \tau_{0 \text { (pris) })=}=\tau_{0 \text { (bas) }} / \tau_{0 \text { (pris) }}=6$, , 
our resolution only affect the description of the transient regime, as explained above. The use of the direct method leads to a response slightly stiffer than with the collocation method but this feature is not proved to be general at the moment. These comparisons show also the pertinence of the affine formulation as compared to the elastic interaction scheme proposed by Weng. Weng's model leads to a stiffer behaviour since no viscous relaxation is accounted for in the phase interactions.

\section{CONCLUSION}

Compared to previous attempts, the affine formulation proposed by Masson and Zaoui [4] presents the advantage to extend the Hill's conception of the non linear self-consistent scheme to viscoelasticity. Consequently, it retajns the viscoelastic coupling of the interactions in a rigorous way. Moreover, no other assumption than the convexity are needed on the local viscoplastic potential.

The simplified resolution presented here is based on an approximation of the inversion of the Laplace-Carson transforms adapted to monotonic loading paths. This leads to a very simple numerical resolution keeping the essential features of the affine formulation. The viscoelastic coupling is retained and the limit behaviours $(t=0$ and $t \rightarrow+\infty)$ are identical to those obtained with a more accurate resolution (collocation method). Pertinent predictions can be obtained for high nonlinear behaviours since the affine viscoplastic model does not coincide with the lower bound as does the tangent model in that case [2].

The first application to nonlinear creep of FCC and HCP polycrystals shows a very good agreement of this direct resolution method with the collocation method, the strain deviation between both being about $1 \%$. The direct method should also be applicable to other monotonic loading paths (relaxation, constant strain rate test...). This will be the matter of future work.

\section{REFERENCES}

1. N. Laws and R. McLaughlin, Proc. Roy. Soc. Lond. (1978) 251-273

2. R. Lebensohn and C. Tomé, Acta Metall. Mater., 41 (1993) 2611-2624

3. R. Masson, PhD Thesis, Ecole Polytechnique, France (1998)

4. R. Masson and A. Zaoui, J. Mech. Phys. Solids, 47 (1999) 1543-1568

5. A. Molinari, Modelling Simul. Mater. Sci. Eng., 7 (1999) 683-697

6. A. Molinari, S. Ahzi and R. Kouddane, Mech. Materials, 26 (1997) 43-62

7. A. Molinari, G.R. Canova and S. Ahzi, Acıa Metall. Mater., 35 (1987) 2983-2994

8. A. Paquin, PhD Thesis, University of Metz, France (1998)

9. Y. Rougier, C. Stoltz and A. Zaoui, C. R. Acad. Sci. Paris (1994) 145-151

10. R.A. Schapery, Proc. U.S. Nat. Congr. Appl. Mech. ASME $4^{\text {th }}(1962) 1075-1085$

11. P. Tumer and C. Tomé, J. Mech. Phys. Solids, 41 (1993) 1191-1211

12. P. Turner, C. Tomé and C. Woo, Phil. Mag. A (1994) 689-711

13. G.J. Weng, J. Appl. Mech, 48 (1981) $41-46$ 